\title{
Secondary bacteraemia in adult patients with prolonged dengue fever
}

\author{
R Premaratna ${ }^{1}$, D Dissanayake ${ }^{3}$, FHDS Silva ${ }^{3}$, M Dassanayake $^{2}$, HJ de Silva ${ }^{1}$
}

(Index words: dengue, bacteraemia)

\begin{abstract}
Introduction Although dengue management guidelines do not advice on use of antibiotics in dengue shock syndrome, unrecognised bactraemia is likely to contribute to morbidity and mortality.
\end{abstract}

Objectives To assess the occurance of secondary bacteraemia in adult patients with prolonged dengue fever.

Methods A prospective study was conducted recruiting patients with confirmed acute dengue infection who had prolonged fever ( $>5$ days). Two sets of blood cultures were taken in such patients prior to institution of antibiotic therapy. Demographic, clinical, haematological and biochemical parameters were recorded. Development of ascites and pleural effusions were detected using ultrasonography.

Results Fourty patients ( $52.5 \%$ males) with a mean age of 29.8 years (SD 13.6) were studied. The average duration of fever was 7.9 days (SD 1.8). Ten patients (25\%) had bacterial isolates in their blood cultures; Staphylococcus aureus $(n=2)$, coliforms $(n=3)$, pseudomonas $(n=1)$ and 4 had mixed growths. The culture positive group had severe body aches at admission and higher fever, third space fluid accumulation, a significant drop in platelets and a higher CRP.

Conclusions A quarter of dengue patients with prolonged fever had a bacterial isolate. Culture positive patients appeared more ill with body aches and had higher degrees of fever during the latter part of the illness. Increased vascular permeability may predispose to bacterial seepage into blood. Although white cell count is not helpful in detecting bacteraemia, low platelet count and elevation of CRP seem to be helpful.

Ceylon Medical Journal 2015; 60: 10-12

\section{Introduction}

Dengue is one of the most important mosquito-borne arbo-viral infections that threatens more than $40 \%$ of the world's population, causing 50-100 million infections every year $[1,2]$. Dengue illness is characterised by a febrile phase, critical phase and the recovery phase.
During the critical phase, there is an increase in vascular permeability resulting in leakage of fluid and plasma or leading to haemorrhage. Although there is no specific treatment, early detection and timely intervention is the key in reducing mortality [2].

The febrile phase of dengue usually lasts for 5-7 days. However some patients continue to have prolonged high fever. Although the critical phase is described as occuring during the immediate defeverscent phase some patients develop the critical phase during the febrile phase $[1,3,4]$. Few studies have shown growth of bacteria in blood cultures suggesting secondary bacteraemia in patients with prolonged dengue fever, probably due to translocation of gut bacteria due to disruption of intestinal mucosal and vascular integrity $[5,6]$. A Japanese case report has shown development of disseminated Candida tropicalis infection in a patient with dengue complicated with liver failure and shock, probably due to translocation of the fungus from the intestine [7]. Furthermore it has been shown that concurrent bacterial infection may cause lipopolysaccharide-mediated enhancement of virus replication and synergistic IFN- $\alpha$ production resulting in disease progression during dengue virus infection [8].

Dengue management guidelines do not recommend use of antibiotics during any stages of dengue infection probably due to sparse evidence of secondary bacteraemia in patients with severe dengue. However, it is important to understand the incidence, risk factors and predictors of bacteraemia in critical illness, because at least a proportion of patients with severe dengue may develop septic shock mimicking or complicating dengue shock resulting in a worse outcome. The aim of the study was to assess the prevalence of bacteraemia and identify risk factors and predictors in dengue patients with prolonged fever.

\section{Methods}

A prospective study was conducted at the University Medical Unit of Colombo North Teaching Hospital, Ragama in 2012. Patients with antigenically or serologically confirmed dengue who were over 18 years of age and having prolonged fever ( $>5$ days) were

Departments of ${ }^{1}$ Medicine, ${ }^{2}$ Microbiology and ${ }^{3}$ University Medical Unit, Faculty of Medicine, University of Kelaniya, Sri Lanka.

Correspondence: RP, e-mail: <ranjanp64@gmail.com>. Received 18 August and revised version accepted 29 December 2014. Competing interests: none declared. 
recruited. Patients who had received antibiotics prior to recruitment to the study were excluded.

An interviewer administered pre-tested questionnaire was developed to obtain demographic, clinical, biochemical and haematological data. They were obtained by direct interview, on site examination and from clinical records where relevant. Data related to fever were obtained from temperature charts maintained by the nursing staff. Body ache was assessed using a visual analogue scale numbered from 0 to 5 , where 0 represented no body ache and 5 represented unbearable body ache as perceived by the patient. Once prolonged fever criteria were fulfilled, two sets of blood cultures were obtained using strict aseptic precautions prior to initiation of antibiotic therapy. Blood cultures were done at the microbiology laboratory, Colombo North Teaching Hospital, Ragama, Sri Lanka. Detection of third space fluid accumulation (TSFA) was done using bedside ultrasonography performed daily. Demographic and clinical characteristics were tabulated between blood culture positive and negative patients. Approval was obtained from the Ethics Committee of the Faculty of Medicine, University of Kelaniya. Informed written consent was obtained from all participants.

\section{Results}

There were 40 patients ( $52.5 \%$ males) mean age was 29.8 years (SD 13.6) (Table 1). Mean duration of fever was 7.9 days (SD 1.8). Ten patients (25\%) had isolates in their blood cultures; Staphylococcus aureus $(\mathrm{n}=2)$, coliforms $(n=3)$, pseudomonas $(n=1)$ and 4 had mixed growths. There was no significant difference in symptoms between the culture positive and negative groups except severity of body ache and fever. Maximum recorded body ache score was more among culture positive patients at admission, and on day 6 of illness. Third space fluid accumulation was more among the culture positive patients (Table 2).

There was a greater drop in platelets and a higher CRP in culture positive patients (Table 3 ). Patients with secondary bacteraemia had a mean stay of 8 days (SD 2.4) and patients without bacteraemia had a mean stay of 6.2 days (SD 1.4).

\section{Discussion}

Table 1. Demographic characteristics

\begin{tabular}{|c|c|c|c|}
\hline & $\begin{array}{l}\text { Total } \\
n=40\end{array}$ & $\begin{array}{l}\text { Culture (+)ve } \\
n=6\end{array}$ & $\begin{array}{c}\text { Culture (-)ve } \\
n=34\end{array}$ \\
\hline $\begin{array}{l}\text { Age in years, } \\
\text { mean (SD) }\end{array}$ & $29.8(13.6)$ & $30.2(15.5)$ & $29.7(13.5)$ \\
\hline Male (\%) & $21 \quad(52.5)$ & $4 \quad(66.7)$ & $17 \quad(50.0)$ \\
\hline
\end{tabular}

Vol. 60, No. 1, March 2015
Table 2. Clinical features of culture positive and culture negative groups

\begin{tabular}{|c|c|c|c|}
\hline Parameter & $\begin{array}{c}\text { Total } \\
\text { Number }=40 \\
n(\%)\end{array}$ & $\begin{array}{c}\text { Culture }(+) v e \\
\text { Number }=6 \\
n(\%)\end{array}$ & $\begin{array}{c}\text { Culture (-)ve } \\
\text { Number }=34 \\
n(\%)\end{array}$ \\
\hline Backache & $40(100)$ & $6(100)$ & $34(100)$ \\
\hline Retro-orbital pain & $18(45)$ & $2(33.3)$ & $16(47.1)$ \\
\hline Abdominal pain & $16(40)$ & $3(50)$ & $13(38.2)$ \\
\hline Vomiting & $14(35)$ & $3(50)$ & $11(32.4)$ \\
\hline Diarrhoea & $1(2.5)$ & $0(0.0)$ & $1(2.9)$ \\
\hline Postural dizziness & $22(55)$ & $5(83.3)$ & $17(50)$ \\
\hline Dyspnoea & $1(2.5)$ & $0(0)$ & $1(2.9)$ \\
\hline \multicolumn{4}{|l|}{$\begin{array}{l}\text { Third space fluid } \\
\text { accumulation }\end{array}$} \\
\hline \multicolumn{4}{|l|}{$\begin{array}{l}\text { Maximum } \\
\text { recorded fever }\end{array}$} \\
\hline mean (SD) & & $102.2^{\circ} \mathrm{F}(1.3)$ & $101.7(1.3)$ \\
\hline \multicolumn{2}{|c|}{$\begin{array}{l}\text { Body aches score } \\
\text { at admission mean (SD) }\end{array}$} & $1.33(0.82)$ & $1.24(0.89)$ \\
\hline \multicolumn{2}{|c|}{$\begin{array}{l}\text { Body aches maximum } \\
\text { score mean }(\mathrm{SD})\end{array}$} & $2.83(0.41)$ & $1.74(1.21)$ \\
\hline \multicolumn{2}{|l|}{$\begin{array}{l}\text { Body aches score } \\
\text { on day } 6 \text { mean (SD) }\end{array}$} & $2.20(0.84)$ & $1.16(1.00)$ \\
\hline
\end{tabular}

Table 3. Maximum values of haematological and biochemical parameters

\begin{tabular}{|c|c|c|}
\hline & $\begin{array}{l}\text { Culture (+)ve } \\
n=6 \\
\text { Mean (SD) }\end{array}$ & $\begin{array}{l}\text { Culture (-)ve } \\
n=34 \\
\text { Mean (SD) }\end{array}$ \\
\hline WBC & $8.5 \times 10^{9} / \mathrm{L}(1.2)$ & $7.1 \times 10^{9} / \mathrm{L}(2.4)$ \\
\hline Neutrophils & $42.0(8)$ & $41.1(9.1)$ \\
\hline Platelets & $\begin{array}{l}36.4 \\
\times 10^{9} / \mathrm{L}(12.2)\end{array}$ & $42.2 \times 10^{9} / \mathrm{L}(21.2)$ \\
\hline PCV (\% rise) & $9.1(17.6)$ & $9.6(19.5)$ \\
\hline AST (\% rise) & $134(219.6)$ & 194.7 (317.9) \\
\hline ALT (\% rise) & $151(272.4)$ & $177.8(330.4)$ \\
\hline Maximum CRP (mg/dl) & $60.0(54.9)$ & $16.1(25.7)$ \\
\hline
\end{tabular}

Of the patients who had prolonged fever, ten had positive cultures $(25 \%)$ and six $(15 \%)$ had a single organism isolate in the blood cultures. Age and sex distribution of culture positive and culture negative samples were comparable.

Culture positive group appeared to be more symptomatic with higher rate of abdominal pain, vomiting, diarrhoea and postural dizziness. The culture positive group reported more severe body ache throughout the illness. Lee et al also found severe bone pain as one of the common clinical manifestations in patients with dual infections similar to our patients [9]. Maximum recorded fever was higher among the culture positive patients. 
Third space fluid accumulation was more among the culture positive patients. It is possible that abnormal vascular permeability, the main cause for dengue shock syndrome, results in seepage of bacteria into blood stream. Three out of the six patients were positive for Enterobacteriaceae similar to findings from other studies [9,10]. This suggests that gut organisms may be responsible for bacteraemia, in severe dengue. This could probably be due to disintegration of intestinal mucosal barriers in dengue haemorrhagic fever patients as reported previously [5,6].

There was no significant difference in white cell (WBC) and neutrophil counts between the two groups. However the maximum recorded CRP was higher among the culture positive group suggesting that it may be more useful than WBC counts to diagnose bacteraemia in these patients.

It has been shown that patients with dual infection are older, have prolonged fever, higher frequencies of acute renal failure, gastrointestinal bleeding, altered level of consciousness, unusual dengue manifestations and dengue shock syndrome [9]. However, except for pro-longed fever and varying degrees of fluid leakage, our patients did not show such manifestations or com- plications. Lee et al in 2012 found bacteraemia as a significant cause of fatality in adult patients with dengue fever [11].

\section{Conclusions}

According to our study, $25 \%$ of dengue patients with prolonged fever had bacteraemia. Culture positive patients appeared to be more ill with body aches and had higher degrees of fever during the latter part of the illness. Although WBC is not helpful in detecting bacteraemia, lower platelet counts and elevation of CRP seem to be helpful. We feel that institution of antibiotics should be considered in similar patients in order to reduce bacteraemia associated complications.

\section{Acknowledgements}

We thank the staff of the University Medical Unit and the Department of Microbiology, Colombo North Teaching Hospital, Ragama, Sri Lanka.

\section{Declaration of Interest}

There are no conflicts of interest.

\section{References}

1. WHO. Dengue and Severe Dengue.WHO Media center. Fact sheet 117. 2014. http://www.who.int/mediacentre/ factsheets/fs117/en/index.html

2. Guzman MG, Kouri G. Dengue: an update. Lancet Infect Dis 2001; 2: 33-42.

3. Premaratna R, Jayasinghe KG, Liyanaarachchi EW, Weerasinghe OM, Pathmeswaran A, de Silva HJ. Effect of a single dose of methyl prednisolone as rescue medication for patients who develop hypotensive dengue shock syndrome during the febrile phase: a retrospective observational study. Int J Infect Dis 2011; 15: e433-4.

4. Premaratna R, Rodrigo KM, Anuratha A, de Alwis VK, Perera UD, de Silva HJ. Repeated dengue shock syndrome and 'dengue myocarditis' responding dramatically to a single dose of methyl prednisolone. Int J Infect Dis 2012; 16: e565-9.

5. Vejchapipat P, Theamboonlers A, Chongsrisawat V, Poovorawan Y. An evidence of intestinal mucosal injury in dengue infection. Southeast Asian J Tropl Med Public Health 2006, 37: 79-82.

6. Limonta D, Capo' V, Torres G, Pe'rez AB, Guzma'n MG. Apoptosis in tissues from fatal dengue shock syndrome. $J$ Clin Virol 2007; 40: 50-4.

7. Suzuki S, Kitazawa T, Ota Y, et al. Dengue hemorrhagic shock and disseminated candidiasis. Intern Med 2007; 46: 1043-6.

8. Chen YC, Wang SY. Activation of Terminally Differentiated Human Monocytes/Macrophages by Dengue Virus: Productive Infection, Hierarchical Production of Innate Cytokines and Chemokines, and the Synergistic Effect of Lipopolysaccharide. J Virol 2002; 76: 9877-87.

9. Lee IK, Liu JW, Yang KD. Clinical characteristics and risk factors for concurrent bacteremia in adults with dengue hemorrhagic fever. Am J Trop Med Hyg 2005; 72: 221-6.

10. Ong A, Sandar M, Chen MI, Sin LY. Fatal dengue hemorrhagic fever in adults during a dengue epidemic in Singapore. Int J Infect Dis 2007; 11: 263- 67.

11. Lee IK, Liu JW, Yang KD. Fatal dengue hemorrhagic fever in adults: emphasizing the evolutionary pre-fatal clinical and laboratory manifestations. PLoS Negl Trop Dis 2012; 6: e1532. 